\title{
STUDY OF INTERSTELLAR EXTINCTION IN THE GALAXY USING LARGE PHOTOMETRIC DATA BASES *
}

\author{
J. Sūdžius and S. Raudeliūnas \\ Astronomical Observatory of Vilnius University, Čiurlionio 29, LT-03100 Vilnius, Lithuania \\ E-mail: jokubas.sudzius@ff.vu.lt
}

Received 20 June 2007; accepted 21 November 2007

\begin{abstract}
The results of the study of variations of the interstellar extinction law in the visible to near infrared in the Milky Way band between galactic latitudes from $-20^{\circ}$ to $+20^{\circ}$ are presented. It is shown that a ratio of total-to-selective extinction, $R_{V}=A_{V} / E_{B-V}$, varies roughly proportionally to the density of obscuring interstellar clouds. It is found that frequency distribution of the ratios $R_{V}$ is trimodal with peaks at 2.42, 2.81, and 3.14.
\end{abstract}

Keywords: interstellar extinction, Milky Way galaxy, stellar photometry

PACS: 97.10.Ri, 98.38.Cp

\section{Introduction}

One of the main sources of information about interstellar dust is the spectral dependence of extinction of a certain interstellar cloud or the so-called interstellar extinction law (ISEL). Knowledge of the ISEL is important not only for the study of physical properties and origin of interstellar dust but also for correct evaluation of the amount of interstellar extinction in the direction of objects obscured by interstellar matter or for recovering their intrinsic spectrophotometric characteristics. Uncertainties or systematic variations of the ISEL may predetermine conclusions based on the data of heavily reddened objects, such as photometric distances, identification of physical groups of stars in colour-colour diagrams, and the age determination of clusters.

Numerous investigations have shown that the ISEL varies from one line of sight to another (see, e.g., Draine [1] and Whittet [2], and references therein). However, the general form of the extinction curve and its features remain the same for many investigated regions of the Milky Way. This fact has been successfully explored by Cardelli et al. [3] who have found that the extinction curve can well be parameterized with a few parameters and that variations of the ISEL can be characterized by one parameter, the ratio of total-toselective extinction, $R_{V}=A_{V} / E_{B-V}$. The ISEL for diffuse interstellar medium may well be described by a

\footnotetext{
* The report presented at the 37th Lithuanian National Physics Conference, 11-13 June 2007, Vilnius, Lithuania.
}

curve with $R_{V}=3.1$, while variations of $R_{V}$ are in the range of 2.2-5.5 [1]. It should be noted that $R_{V}$ can also be treated as a rough indicator of interstellar dust grain size: regions with lower $R_{V}$ have smaller grains, while regions with higher $R_{V}$ have larger grains. In addition, many authors have determined that the ISEL in the near infrared (NIR, $1 \mu \mathrm{m}<\lambda<5 \mu \mathrm{m}$ ) is invariant and that extinction curve in this region may well be represented by a power law, $A_{\lambda} \sim \lambda^{-\beta}$, where $\beta=1.8$ according to Martin \& Whittet [4]. This value of $\beta$ implies that the colour excess ratio $E_{J-H} / E_{H-K}=1.8$. Some authors, however, report that this ratio ranges from 1.3 to 2.1 for different regions of the Milky Way (see, e. g., [5-9]). There is no definite answer whether these differences arise from differing properties of interstellar dust, or from inhomogeneous photometric data.

It is important to note that many investigations of the ISEL in the NIR made up to the end of the 20th century were based on photometry of a comparatively small number of stars carried out in slightly differing photometric systems. This could lead to false conclusions about differences or similarities of the ISEL in different regions of the Milky Way. Fortunately, with the beginning of the 21 st century a number of new global photometric surveys in the IR emerged. Advantages of large surveys compared with small data sets obtained using different photometric systems and applying different methods are evident. They cover large areas of the sky and their data are more homogeneous 
than those selected from a variety of small catalogues. Such surveys coupled with homogeneous $B V$ photometry enable researchers to carry out more precise and thorough investigations and to draw more coherent picture of variations of the ISEL in the galaxy.

The aim of the present work was to investigate possible variations of the ISEL in the whole Milky Way on the basis of 2MASS survey [10] and Tycho-2 photometry [11]. It is the extension of our previous work [12]. Present work is based on larger number of stars and includes higher galactic latitudes.

\section{The data}

Our investigation embraced the whole Milky Way between galactic latitudes $-20^{\circ}<b<20^{\circ}$ since it was not expected to find many reddened stars at higher galactic latitudes. Analysis of variations of the ISEL is based on the colour difference method. For that purpose we compiled a catalogue of $B_{T}, V_{T}, J, H$, and $K$ magnitudes of stars with known spectral types. Magnitudes $B_{T}$ and $V_{T}$ (mean wavelengths 0.42 and $0.52 \mu \mathrm{m}$, respectively) were taken from Tycho-2 catalogue [11], while magnitudes $J, H$, and $K$ (mean wavelengths $1.24,1.66$, and $2.16 \mu \mathrm{m}$, respectively) from the catalogue 2MASS [10]. Sources of spectral types of stars were compilations of Wright et al. [13], Kharchenko [14], and Skiff [15]. We selected stars that were located in the Milky Way between galactic latitudes $-20^{\circ}<$ $b<20^{\circ}$ and that were "normal" in their spectral class, the latter being in the range from O to G7. Stars excluded from further analysis were as follows:

- Stars with observational errors in colour indices $B_{T}-V_{T}$ larger than 0.05 mag or with negative colour excesses.

- Stars that were recorded as variable stars.

- Double stars.

- Stars listed as having emission lines.

- Stars, for which peculiar colours were recorded.

The final compilation contained about 94000 stars. Procedure of calculation of colour excess ratios was similar to that used in our previous paper [12]. Colour excesses $E_{B-V}, E_{V-J}, E_{V-H}, E_{V-K}, E_{J-H}$, and $E_{H-K}$ were evaluated using intrinsic colour indices from Straižys [16]. These colour excesses enabled us to compute colour excess ratios $E_{V-J} / E_{B-V}$, $E_{V-H} / E_{B-V}$, and $E_{V-K} / E_{B-V}$ that were sensitive to variations of the ISEL in the optical and IR regions of

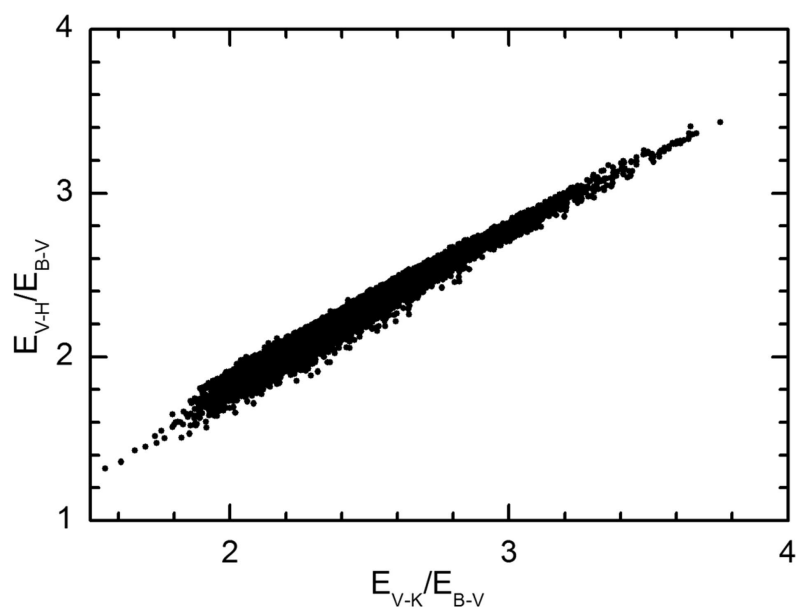

Fig. 1. Relationship between colour excess ratios $E_{V-K} / E_{B-V}$ and $E_{V-H} / E_{B-V}$.

the spectrum, and $E_{J-H} / E_{H-K}$, which should show variations in the NIR.

\section{Results and discussion}

The main indicator of variations of the ISEL is the colour excess ratio $E_{V-K} / E_{B-V}$, which has well established relationship with the ratio $R_{V}$ (see, e.g., $[2,17])$. Since the passbands $J$ and $H$ are also located in the NIR, variations of the $E_{V-J} / E_{B-V}$ and $E_{V-H} / E_{B-V}$ should mimic variations of the ratio $E_{V-K} / E_{B-V}$, thus confirming the reality of changes of the ISEL. As an example we present Fig. 1, which demonstrates good correlation between ratios $E_{V-K} / E_{B-V}$ and $E_{V-H} / E_{B-V}$. Similar correlation was obtained for the ratios $E_{V-K} / E_{B-V}$ and $E_{V-J} / E_{B-V}$ as well. Distribution of the $E_{V-K} / E_{B-V}$ values in the plane of galactic longitudes and latitudes is shown in Fig. 2. Since the average error of this ratio is of the order of 0.07 , we can conclude that these variations are statistically significant. Variations of the $E_{V-K} / E_{B-V}$ are in the range from 1.9 to 3.65 , which corresponds to the range of the ratios $R_{V}$ from 2.0 to 3.8 . We did not get extremely large values of the $E_{V-K} / E_{B-V}$ due to smoothing effect, which is determined by our method of computation of the colour excess ratios. Therefore, we were not able to identify compact regions (individual associations and / or clusters) with extremely large colour excess ratios $E_{V-K} / E_{B-V}$. It seems that variations of the $E_{V-K} / E_{B-V}$ are quite irregular and it is difficult to select its most typical value for the whole Galaxy. Regions with higher values of the $E_{V-K} / E_{B-V}$ concentrate towards the galactic equator, while regions with the lowest ratios $E_{V-K} / E_{B-V}$ are mostly found 


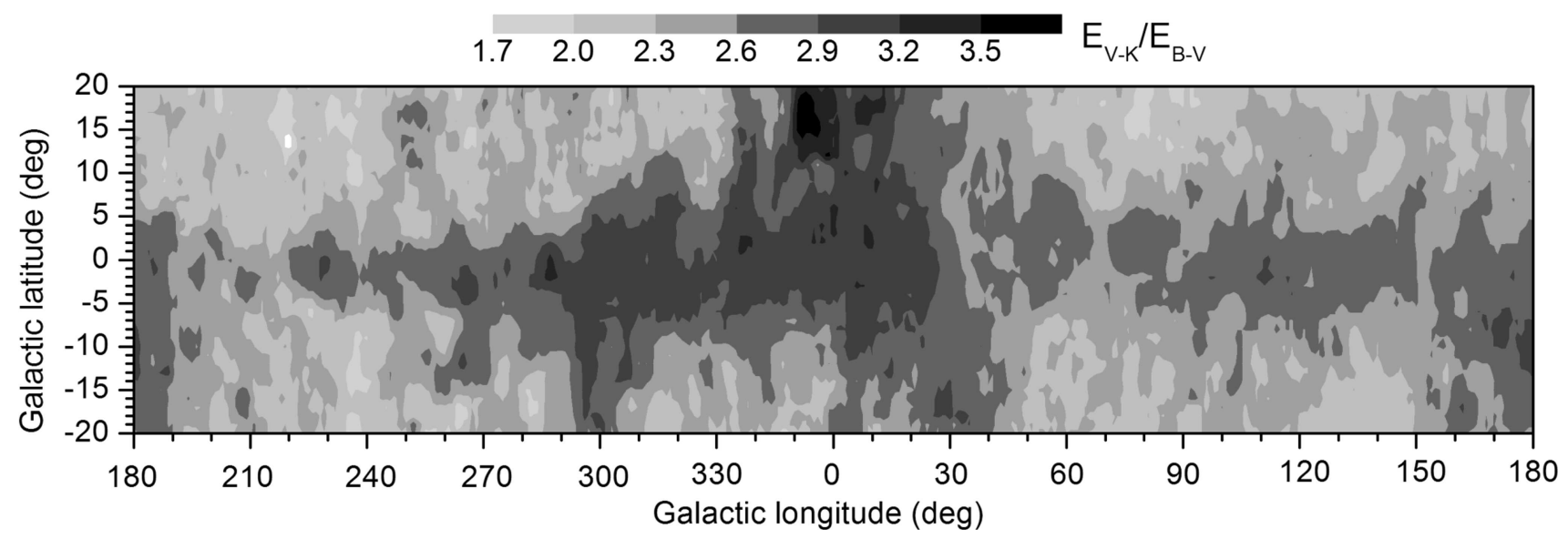

Fig. 2. Distribution of the ratios $E_{V-K} / E_{B-V}$ in galactic coordinates. Darker areas correspond to larger values of the ratios $E_{V-K} / E_{B-V}$ (see panel).

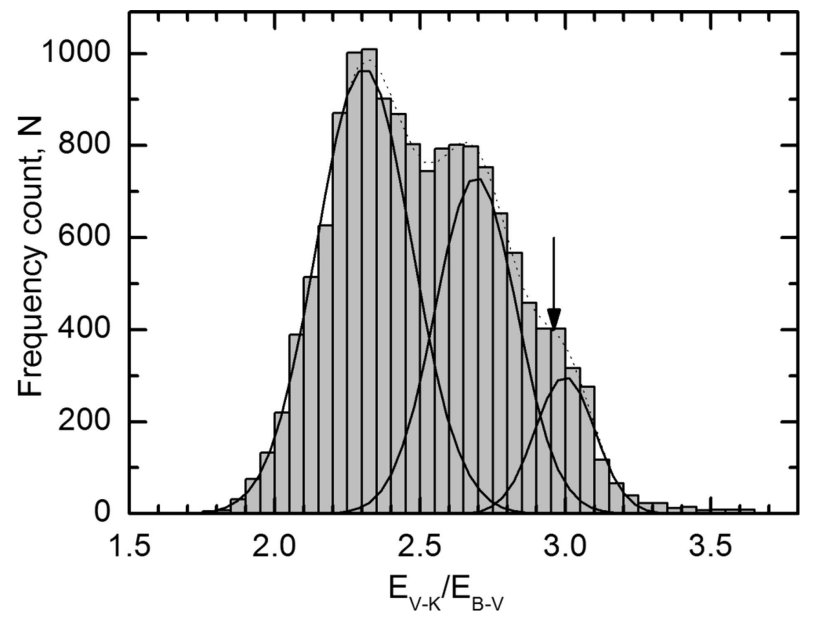

Fig. 3. Frequency histogram of the ratios $E_{V-K} / E_{B-V}$. Overlaid on the histogram is the trimodal Gaussian distribution. The vertical arrow indicates the value of the ratio $E_{V-K} / E_{B-V}$ corresponding to $R_{V}=3.1$.

at large galactic latitudes. Also, the ISEL is not uniform towards different directions of the galactic equator. The wide region of large $E_{V-K} / E_{B-V}$ values stretches in the environs of the Galactic Centre, and the region of low ratios $E_{V-K} / E_{B-V}$ lies in the direction of galactic longitudes $210^{\circ}-220^{\circ}$. The latter line of sight corresponds to the direction of the inter-arm region where lower density of interstellar matter is expected. Area of large ratios $E_{V-K} / E_{B-V}$ around galactic longitude of $355^{\circ}$ and latitude of $17^{\circ}$ is located in the region of dark $\rho$ Ophiuchi clouds. Consequently, our results confirm the conclusion of earlier authors that the ratio $R_{V}$ varies roughly proportionally to the density of obscuring interstellar clouds. Frequency distribution of the ratios $E_{V-K} / E_{B-V}$ is shown in Fig. 3. It is evident that this histogram is rather asymmetric and has three distinct peaks. This histogram was fitted by compos- ite Gaussian distribution that was derived by adding three single Gaussian distributions. Maximums of distributions are located at the ratios $E_{V-K} / E_{B-V} 2.31$, 2.69 , and 3.00, which correspond to the $R_{V}$ values of $2.42,2.81$, and 3.14 , respectively. The average value of $R_{V}=3.1$ established for diffuse interstellar clouds is close to the third maximum. It seems that distribution of the ratios $E_{V-K} / E_{B-V}$ is trimodal. If this is the case, then we should be able to separate three different families of clouds, each of which is characterized by a particular composition and / or size distribution of dust grains. Solution of this problem requires further studies of properties of the ISEL and interstellar dust.

Ratios $E_{J-H} / E_{H-K}$ computed in the present work have shown larger diversity of values than expected according to cited literature [5-9]. We assume that this diversity is not real but caused by inaccuracies of intrinsic colour indices. In order to make more definite conclusion about variations of the ratio $E_{J-H} / E_{H-K}$ further investigations are needed.

\section{Acknowledgements}

This publication makes use of data products from the Two Micron All Sky Survey (2MASS), which is a joint project of the University of Massachusetts and the Infrared Processing and Analysis Center, California Institute of Technology, funded by the NASA and the National Science Foundation, USA. This research has also made use of the Simbad database, operated at the Centre de Données astronomiques de Strasbourg, France. 


\section{References}

[1] B.T. Draine, Interstellar dust grains, Ann. Rev. Astron. Astrophys. 41, 241 (2003).

[2] D.C.B. Whittet, Dust in the Galactic Environment, 2nd ed. (Institute of Physics Publishing, Bristol, 2003).

[3] A. Cardelli, G.C. Clayton, and J.S. Mathis, The relationship between infrared, optical, and ultraviolet extinction, Astrophys. J. 345, 245 (1989).

[4] P.G. Martin and D.C.B. Whittet, Interstellar extinction and polarization in the infrared, Astrophys. J. 357, 113 (1990).

[5] T.J. Jones and A.R. Hyland, New results on interstellar reddening in the near infrared, Mon. Not. R. Astron. Soc. 192, 359 (1980).

[6] D.S. Davis, H.P. Larson, and R. Hofmann, $\mathrm{H}_{2}$ spectroscopy as an agent for extinction determinations: The near-infrared curve for the Orion molecular cloud, Astrophys. J. 304, 481 (1986).

[7] S.J. Kenyon, E.A. Lada, and M. Barsony, The nearinfrared extinction law and limits on the pre-mainsequence population of the $\rho$ Ophiuchi dark cloud, Astron. J. 115, 252 (1998).

[8] M. Gómez and S.J. Kenyon, A near-infrared imaging survey of the Chamaeleon I dark cloud, Astron. J. 121, 974 (2001.)

[9] G. Racca, M. Gómez, and S.J. Kenyon, A near-infrared imaging survey of Coalsack Globule 2, Astron. J. 124, 2178 (2002).

[10] R.M. Cutri, M.F. Skrutskie, S. van Dyk, C.A. Beichman, J.M. Carpenter, T. Chester, L. Cambresy, T. Evans, J. Fowler, J. Gizis, E. Howard, J. Huchra,
T. Jarrett, E.L. Kopan, J.D. Kirkpatrick, R.M. Light, K.A. Marsh, H. McCallon, S. Schneider, R. Stiening, M. Sykes, M. Weinberg, W.A. Wheaton, S. Wheelock, and N. Zacarias, The 2MASS All-Sky Catalog of Point Sources (University of Massachusetts and Infrared Processing and Analysis Center, IPAC/ California Institute of Technology, 2003) [Vizier on-line data catalogue II/246, 2003].

[11] E. Høg, C. Fabricius, V.V. Makarov, S. Urban, T. Corbin, G. Wycoff, U. Bastian, P. Schwekendiek, and A. Wicenec, The Tycho-2 catalogue of the 2.5 million brightest stars, Astron. Astrophys. 355, L27 (2000) [Vizier on-line data catalogue $\mathbf{I} / \mathbf{2 5 9}, 2000$ ].

[12] J. Sūdžius and S. Raudeliūnas, Variations of the ratio of total-to-selective extinction in the galaxy, Baltic Astronomy 12, 520 (2003).

[13] C.O. Wright, M.P. Egan, K.E. Kraemer, and S.D. Price, The Tycho-2 spectral type catalog, Astron. J. 125, 359 (2003) [Vizier on-line data catalogue III/231, 2003].

[14] N.V. Kharchenko, All-sky compiled catalogue of 2.5 million stars, Kinemat. Physic. Celestial Bodies 17, 409 (2001) [Vizier on-line data catalogue I/280A, $2001]$.

[15] B.A. Skiff, Catalogue of Stellar Spectral Classifications (Lowell Observatory, 2005) [Vizier on-line data catalogue III/233B, 2005].

[16] V. Straižys, Multicolor Stellar Photometry (Pachart Publishing House, Tucson, Arizona, 1992).

[17] E.L. Fitzpatrick, Correcting for the effects of interstellar extinction, Publ. Astron. Soc. Pacific 111, 63 (1999).

\title{
DIDELIU FOTOMETRINIŲ DUOMENU BAZIU PANAUDOJIMAS TARPŽVAIGŽDINEI EKSTINKCIJAI GALAKTIKOJE TIRTI
}

\author{
J. Sūdžius, S. Raudeliūnas
}

Vilniaus universiteto Astronomijos observatorija, Vilnius, Lietuva

\section{Santrauka}

Ištyrinètos tarpžvaigždinès ekstinkcijos dèsnio variacijos priklausomai nuo galaktinès ilgumos ir platumos. Rasta, kad daugiklis $R_{V}=A_{V} / E_{B-V}$, apibūdinantis tarpžvaigždinès ekstinkcijos dèsni, kinta maždaug proporcingai tarpžvaigždinių debesų tankiui. Pagal šio daugiklio verčiu pasiskirstymą nustatytos trys charakteringos jo vertès: $2,42,2,81$ ir 3,14 . 